Artículo original

\title{
Muestra de saliva para diagnóstico de SARS-CoV-2 por RT-qPCR en población ambulatoria
}

\author{
DOI 10.5377/alerta.v4i2.11476 \\ Noé Rigoberto Rivera ${ }^{1}$, Carlos Alexander Ortega Pérez ${ }^{2^{*}}$, Xochitl Sandoval López ${ }^{3}$, Carlos Enrique Hernández \\ Ávila ${ }^{4}$ \\ 1. Unidad de Investigaciones Científicas, Facultad de Medicina, Universidad de El Salvador, El Salvador C.A. \\ 2. Sección de Virología, Facultad de Medicina, Universidad de El Salvador, El Salvador C.A. \\ 3. Instituto Nacional de Salud, Ministerio de Salud, El Salvador C.A. \\ 4. Unidad de Gobernanza, Instituto Nacional de Salud, El Salvador C.A. \\ *Correspondencia \\ $\square$ carlos.ortega@ues.edu.sv \\ 1. (D) 0000-0001-9553-0554 \\ 2. (D) $0000-0003-1612-8336$ \\ 3. (D) $0000-0002-0988-1313$ \\ 4. (D) $0000-0002-1230-0102$
}

\section{G}

ACCESO ABIERTO

Saliva sample selfsampling for the

diagnosis of SARS-

CoV-2 by RT-qPCR in an

outpatient population

\section{Citación recomendada:}

Rivera NR, Ortega CA, Sandoval

López X, Hernández Ávila

CE. Muestra de saliva para

diagnóstico de SARS-CoV-2

por RT-qPCR en población

ambulatoria. Alerta.

2021:4(2):38-45. DOI 10.5377/

alerta.v4i2.11476

\section{Recibido:}

2 de mayo 2021

\section{Aceptado:}

20 de mayo 2021

\section{Publicado:}

1 de junio 2021

\section{Contribución de autoría:} NRR', CAOP 2 XSL ${ }^{3}, C E H A^{4}$.

Escritura del manuscrito. NRR ${ }^{1}$ $\mathrm{CAOP}^{2}$, diseño del estudio, procesamiento de las muestras y análisis de los datos en las plataformas bioinformáticas.

\section{Conflicto de intereses:}

Los autores declaran no tener conflictos de interés en el estudio ni haber recibido patrocinio de instituciones privadas.

\begin{abstract}
Resumen
Introducción. En El Salvador a la fecha, la técnica utilizada por el sistema nacional de salud para la obtención de la muestra para realizar PCR para SARS-CoV-2 es hisopado nasofaríngeo, diferentes investigadores han descrito la muestra de saliva como una muestra biológica útil para la detección de SARS-Cov-2, por esta razón se observa la oportunidad de aplicarla como una alternativa disponible para el diagnóstico de esta enfermedad. Objetivo. Evaluar la autotoma de muestra de saliva y secreción nasofaríngea por pacientes no hospitalizados como una alternativa de menor riesgo biológico y de menor costo que los hisopados nasofaríngeos convencionales. Metodología. Se procesaron las muestras de una mezcla de saliva y secreción faríngea obtenida por carraspeo autotomada por el paciente; la amplificación se realizó por RT-qPCR de los genes Ey RdRp. Las muestras positivas se reevaluaron desde su extracción para confirmar la estabilidad de material genético de SARS-CoV-2 en la saliva y secreción nasofaríngea. Resultados. El promedio de resultados positivos fue de 7,05 por cada 100 pruebas COVID-19 realizadas con hisopado, este resultado es similar al $8 \%$ de positividad durante el mismo período de estudio utilizando como muestra saliva y secreción faríngea autotomada por el paciente. Las ocho muestras positivas mantuvieron su reactividad para los genes $E$ y RdRp al primer, tercer y quinto mes posdiagnóstico inicial para los dos protocolos utilizados. De igual forma, los eluidos de ARN positivos iniciales se mantuvieron positivos al primer, tercer y quinto mes. Conclusión. La muestra de saliva y secreción faríngea y su utilización para el diagnóstico de infección por SARSCoV-2 podría ser una alternativa de bajo costo, no invasiva, al menos de igual utilidad que el hisopado nasofaríngeo para el estudio de población sintomática ambulatoria o con exposición a nivel comunitario.
\end{abstract}

Palabras clave

SARS-CoV-2, COVID-19, PCR, saliva

\begin{abstract}
Introduction. Currently, the detection of SARS-CoV-2 cases in El Salvador has been carried out through RT-PCR by the nasopharyngeal swab sample. Researchers had described the saliva as a biological sample useful for detection of SARSCov-2, therefore an opportunity to use it as a feasible alternative for diagnostic. Objective. To evaluate the self-supplied sample of saliva and nasopharyngeal secretion by non-hospitalized patients as an alternative of lower biological risk and less expensive to nasopharyngeal swab for the diagnosis of SARS-CoV-2. Methodology. Patient samples that met the inclusion criteria were processed, amplification was carried out by two protocols already standardized by RT-qPCR of the E and RdRp genes, two of the positive samples by New Generation Sequencing (NSG) for confirmation diagnosis. Positive samples were re-evaluated from their extraction and amplification at the first, second, and fifth months after diagnosis to evaluate the stability of the SARS-CoV-2 genetic material in saliva and nasopharyngeal secretion. Results. The average of positives per 100 tests in El Salvador in the month of November 2020 was approximately 7,05 for every 100 COVID-19 tests performed with swabs, this result is similar to the $8 \%$ positivity during the same month of the present study, using as sample a mixture of saliva and pharyngeal secretion self-taken by the patient. The eight positive samples maintained their positivity for the E and RdRp genes at the first, third, and fifth months after the initial diagnosis for both protocols. Similarly, the initial positive Ribonucleic Acid (RNA) eluates remained positive at the first, third, and fifth months. Conclusion. The sample of saliva and pharyngeal secretion and its use for the diagnosis of infection by SARS-CoV-2 could be a low-cost, non-invasive alternative with the same utility as the nasopharyngeal swab for the study of the symptomatic outpatient population or with a community level exposure. Likewise, it could be used for mass screening or sentinel surveillance in settings with limited resources.
\end{abstract}

Keywords

SARS-CoV-2, COVID-19, PCR, saliva, RdRp 


\section{Introducción}

De los coronavirus, dos virus altamente patógenos, Coronavirus del Síndrome Respiratorio Agudo Grave (SARS-CoV) y el Síndrome Respiratorio del Medio Oriente (MERS-CoV) causan síndrome respiratorio severo en humanos y el SARS-CoV-2 causa la actual pandemia desde inicios del 20201,2,3. Desde marzo de 2020 se reportan los casos de infección por SARS-CoV-2 en El Salvador', utilizando RT-qPCR y como muestra, la secreción nasofaríngea recomendada en las orientaciones provisionales para pruebas diagnósticas de SARS-CoV-2 ${ }^{5}$. El 7 de marzo de 2021 el Ministerio de Salud reportaba 60800 casos positivos y 1907 fallecidos $^{4}$ para esta misma fecha el Centro de Ciencia e Ingeniería de Sistemas (CSSE) de la Universidad Johns Hopkins reportaba a nivel mundial 116822839 casos y 2593073 fallecidos por SARS-CoV-26. La Organización Mundial de la Salud reportó el 8 de marzo de 2021 que se habían aplicado 249160837 dosis de vacunas a nivel mundial ${ }^{7}$, se estima que entre el $60 \%$ al $70 \%$ de las personas necesitan ser inmunizadas para lograr inmunidad colectiva-10. A pesar de la implementación del mecanismo COVAX la inmunidad colectiva no se logrará durante $2021^{11,12}$, por lo que es importante continuar con la vigilancia epidemiológica genómica mediante el diagnóstico molecular y secuenciación de SARS-CoV-2.

\section{Estructura y genoma de SARS-CoV-2}

Los coronavirus son virus envueltos con un genoma ARN monocatenario de sentido positivo de aproximadamente 26-32 kilobases, forman partículas esféricas o pleomórficas envueltas de 100 a $160 \mathrm{~nm}$ de diámetro ${ }^{13}$. Su genoma codifica para 14 marcos de lectura abierto (ORF del inglés Open Reading Frame). En el extremo 5', los primeros son los ORF1a y ORF1ab que codifican para poliproteínas que se procesan autoproteolíticamente en 16 proteínas no estructurales, este complejo replicasa-transcriptasa consta de múltiples enzimas. En el extremo 3' otros ORFs en el tercio distal del genoma codifican para al menos cuatro proteínas estructurales principales: proteína de espícula (S), glicoproteína de membrana (M), envoltura (E) y nucleocápside $(\mathrm{N})$, además nueve factores accesorios putativos ${ }^{14-17}$.

\section{RT-qPCR para diagnóstico de SARS-CoV-2}

En una revisión sistemática se evaluaron un total de 67 pruebas de laboratorio diferen- tes utilizando la RT-qPCR como estándar de referencia, encontrándose una heterogeneidad considerable entre las pruebas, los valores de umbral y los contextos en que se aplicaron ${ }^{18}$. La RT-qPCR para el diagnóstico de COVID-19 se realiza con hisopos nasofaríngeos u otras muestras del tracto respiratorio superior, incluidos hisopos de garganta o, más recientemente, saliva. Se utiliza una variedad de objetivos dirigidos a uno o más genes de la envoltura (Env), nucleocápside (N), espícula (S), ARN polimerasa dependiente de ARN (RdRp) y ORF1, la sensibilidad y capacidad de detección del SARS-CoV-2 varía dependiendo del gen amplificado ${ }^{20}$.

En la infección sintomática por COVID-19, el ARN viral en frotis nasofaríngeo medido por el umbral del ciclo (Ct) es detectable desde el día 1 de los síntomas y alcanza su máximo dentro de la primera semana del inicio de los síntomas ${ }^{19,21}$. La evolución temporal de la positividad y la seroconversión de la PCR puede variar en niños y otros grupos, incluida la gran población de individuos asintomáticos. La positividad de la RTqPCR disminuye más lentamente en el esputo y aún puede ser positiva después que los hisopados nasofaríngeos sean negativos. En 205 pacientes con infección confirmada por COVID-19, la positividad fue más alta en las muestras de lavado broncoalveolar (93\%), seguida de esputo (72\%), frotis nasal (63\%) y frotis faríngeo (32\%), la persistencia de la RT-qPCR positiva en el esputo y las heces fue similar ${ }^{19,22}$. En un ensayo de evaluación de la sensibilidad de la RT-qPCR se encontró mayor sensibilidad del gen $\mathrm{E}$ y del gen RdRp (5,2 y 3,8 copias por reacción respectivamente con una probabilidad de detección del 95 \%). En el presente estudio la detección del gen $\mathrm{N}$ también dio resultados positivos, pero mostro menor sensibilidad ${ }^{20}$.

\section{Muestras para el diagnóstico de infección de SARS-CoV-2}

La toma del hisopado nasofaríngeo causa estornudos, tos y dolor nasofaríngeo en la mayoría de los casos, los pacientes generan gotitas o partículas de aerosol que son peligrosas para los trabajadores sanitarios que recogen estas muestras, esta exposición del personal sanitario dificulta la toma de muestras ${ }^{5}$.

Una alternativa no invasiva y confiable sería la detección de SARS-CoV-2 en saliva que se compara con las pruebas de hisopados convencionales, obteniendo resultados comparables entre las muestras $23,24,25$. Sin embargo la OMS insta a que antes de recurrir a otros métodos de obtención de muestras de líquidos respiratorios o bucales, el 
método de muestreo debe ser primero validado en el laboratorio para los grupos de pacientes previstos, recopilando sus propios datos de rendimiento en relación con el método local de recogida de muestras ${ }^{5}$.

En análisis de muestras emparejadas de saliva de garganta profunda e hisopado nasofaríngeo para diagnóstico de infección por SARS-CoV-2 mediante RT-qPCR se encontró una tasa de detección de las muestras de saliva de garganta profunda de $53,7 \%$ y de hisopado nasofaríngeo de $47,4 \%$, ambas muestras fueron comparables para detección del SARS-CoV-223. Otra investigación sobre el diagnóstico de infección por SARS-CoV-2 mediante el uso de saliva demostró la capacidad de detectar el SARS-CoV-2 por RT-qPCR en la saliva almacenada a $4{ }^{\circ} \mathrm{C}$ durante un período de 1 semana, mostrándose que este método es útil para el diagnóstico del SARS-CoV-226. En protocolos de amplificación isotérmica para la detección de SARS-CoV-2 para muestras de hisopos de nariz y garganta, se obtuvieron resultados comparables con el método estándar, logrando aplicar el mismo protocolo en la muestra de saliva con resultados comparables de los casos confirmados ${ }^{27}$.

La precisión diagnóstica en saliva es similar a la del hisopado nasofaríngeo con una sensibilidad combinada en saliva del 83,2 \% y especificidad combinada del 99,2\%; con sensibilidad combinada para hisopado nasofaríngeo del $84,8 \%$ y especificidad del 98,9\% en los análisis primarios. Los resultados fueron similares en los análisis secundarios, sugiriendo que la precisión diagnóstica en saliva es similar con hisopado nasofaríngeo ${ }^{28}$. En otro estudio comparativo de saliva e hisopado nasofaríngeo y faríngeo estándar se encontró una sensibilidad y especificidad de la muestra de saliva para la RT-qPCR de $84,2 \%(95 \%$ Cl 60,4 \% - 96,6\%) y $98,9 \%$ (95\% Cl 96,1 \% - 99,9\%), respectivamente. En el análisis de concordancia entre las dos muestras demostró una concordancia observada del 97,5\% (coeficiente k 0,851; IC del $95 \%$ : 0,723-0,979; $p<0,001)$, concluyendo que la saliva podría ser una muestra alternativa para el diagnóstico de COVID-1929.

\section{Efecto de ribonucleasa salival en el análisis de las muestras de saliva}

Para analizar esta posibilidad diagnostica un grupo investigador realizó un estudio en el Hospital Universitario de Keio (Tokio, Japón), se obtuvieron frotis nasofaríngeos y muestras de saliva, simultáneamente, de pacientes ingresados en tratamiento con COVID-19 y del personal universitario que presentaban síntomas que sugirieran in- fecciones virales agudas. Las muestras de hisopos nasofaríngeos fueron recolectadas por personal médico capacitado y las muestras de saliva fueron recolectadas por los propios pacientes. En general, las muestras de hisopos nasofaríngeos y de saliva mostraron una concordancia del 86,7 \% con un coeficiente kappa de 0,625. Aunque las muestras recolectadas mucho después del inicio de los síntomas mostraron resultados discordantes, los obtenidos dentro de los 10 días desde el inicio de los síntomas $(n=140)$ presentaron una concordancia del 96,4\% entre ambos tipos de muestras (coeficiente kappa: 0,883), indicando que la saliva, especialmente recolectada dentro de los 10 días posteriores al inicio de los síntomas, puede sustituir las muestras de frotis nasofaríngeos. El almacenamiento a largo plazo de las muestras de saliva en este estudio no afectó los resultados de la prueba incluso en presencia de ribonucleasa salival. Esto sugiere que las muestras de saliva recolectadas incluso en las casas de los pacientes pueden transportarse a laboratorios distantes sin perder sensibilidad. La fragmentación del ARN por ribonucleasa salival podría resultar en una pérdida de viabilidad, pero preservar la detectabilidad por sonda sin descomposición durante días incluso a $25^{\circ} \mathrm{C}$. Los valores del umbral del ciclo (CT del inglés Cycle Threshold) CT de las muestras de saliva no fluctuaron con el tiempo ex vivo ${ }^{30}$.

Las RT-qPCR para SARS-CoV-2 con saliva recolectada en una fase aguda son tan precisas como las que usan hisopado nasofaríngeo, y el almacenamiento de muestras de saliva a temperatura ambiente por varias horas, no afecta los resultados de la prueba. De igual forma se ha demostrado la posibilidad de análisis de grupos de muestras de saliva para cribados masivos ${ }^{30,31}$

Para la muestra de saliva y secreción nasofaríngea deben evaluarse las posibles diferencias entre la saliva obtenida por saliva pasiva o materiales absorbentes y por carraspeo. La saliva de los pacientes infectados puede contener virus que pueden permitir la transmisión por el aire y también por gotitas orales y por lo tanto, debe manipularse con cuidado. Las ventajas de las muestras de saliva son: el paciente la recoge fácilmente, no requiere de personal especializado para su manejo reduciendo los riesgos biológicos, puede tomarse directamente en cualquier lugar requerido ${ }^{32,33}$.

Otro grupo investigador detectó más copias de ARN del SARS-CoV-2 en muestras de saliva (logaritmo de copias promedio por mililitro de 5,58; intervalo de confianza del $95 \%, 5,09$ a 6,07) que en muestras de hisopado nasofaríngeo (media logarítmica de co- 
pias por mililitro, 4,93; IC del $95 \%, 4,53$ a 5,33). Además, un mayor porcentaje de muestras de saliva que las muestras de hisopos nasofaríngeos fueron positivas hasta 10 días después del diagnóstico de Covid-19. De 1 a 5 días después del diagnóstico, el 81 \% (IC del $95 \%, 71$ a 96) de las muestras de saliva fueron positivas, en comparación con el 71 \% (IC del $95 \%, 67$ a 94) de las muestras de hisopado nasofaríngeo. Los hallazgos sugieren que las muestras de saliva y las muestras de frotis nasofaríngeos tienen al menos una sensibilidad similar en la detección del SARS-CoV-2 ${ }^{34}$.

\section{Metodología}

Diseño del estudio exploratorio. Se incluyeron a mayores de 21 años con o sin antecedentes de fiebre o enfermedades respiratorias agudas, otros signos o síntomas compatibles con COVID-19 dentro de los 14 días previos a la toma de muestra, historial de contacto con caso confirmado o sospechoso. Todo se sistematizó con lista de verificación de autollenado rápido.

El día de la recolección de la muestra se dieron recomendaciones a los participantes para que una hora antes de la toma no ingirieran alimentos sólidos, ni líquidos, excepto agua; no masticar golosinas o chicles, no fumar o utilizar vaporizador con o sin sabor; no utilizar algún tipo de spray bucal; no lavarse los dientes, ni utilizar enjuagues bucales o hilo dental y no utilizar productos cosméticos como lápiz labial, bálsamos o cremas labiales.

Se recolectaron 100 muestras de $2 \mathrm{ml}$ como volumen mínimo de una mezcla de saliva y secreción faríngea por carraspeo autotomada por el paciente, utilizando tubos de polipropileno de $14 \mathrm{ml}$ vacíos y estériles (sin medio de transporte), la recolección fue realizada en espacios abiertos con distanciamiento mayor a 5 metros entre cada persona. Las muestras recolectadas fueron trasladadas en termo hielera hacia el laboratorio.

\section{Procesamiento de la muestra}

Previo al procesamiento, las muestras se centrifugaron a 2000 RPM por 5 segundos y sin abrir los tubos se procedió a inactivar el virus a $65^{\circ} \mathrm{C}$ por 30 minutos buscando una reducción mayor de 4 log del coronavirus infectante, esta temperatura se determinó de acuerdo a resultados de diversos estudios de inactivación de SARS-CoV-2 por temperatura ${ }^{35-37}$.

La extracción se realizó con el kit PureLink Viral RNA/DNA Mini Kit Invitrogen de ThermoFisher Scientific ${ }^{\circledR}$. Los eluidos fueron con- servados a $-80^{\circ} \mathrm{C}$ después de la extracción. La concentración de ARN fue determinada por fluorometría con Quantus fluorometer, Promega ${ }^{\oplus}$ siguiendo las instrucciones del fabricante.

Se realizó amplificación por $\mathrm{RT}$-qPCR de los genes E y RdRp con dos protocolos: Charité, Berlin /EVAg, con la AgPath-ID OneStep RT-PCR de ThermoFisher Scientific ${ }^{\oplus}$ y con PowerChek 2019-nCoV Real-time PCR $\mathrm{kit}^{\oplus}$ (https://www.fda.gov/media/140069/ download). Las amplificaciones se realizaron en el QuantStudio 5 Real-Time PCR instrument de Applied Biosystems ${ }^{\oplus}$.

\section{Positividad de la muestras}

Se consideraron como muestras positivas a SARS-CoV-2 si los valores de CT de los genes E y RdRp eran $\leq 39$, y negativo cuando los valores de $\mathrm{CT}$ de ambos objetivos eran $>39$, siendo 39 el punto de corte. Se estableció que en las muestras con resultados de CT discordantes entre los genes investigados, por ejemplo, muestras con gen $\mathrm{E}$ con un valor de CT de $\leq 39$ y gen RdRp con un valor de CT de $>39$ o viceversa, se repetiría el ensayo y si la discordancia persistía en esos valores de CT, estas se darían como negativas.

Las muestras positivas se almacenaron a $-80{ }^{\circ} \mathrm{C}$ en alícuotas de $250 \mu \mathrm{L}$ para evaluar con el mismo protocolo de extracción y amplificación la viabilidad de positividad y mantenimiento o modificación del CT de las muestras al $1^{\circ}, 3^{\circ}$ y $5^{\circ}$ mes pos diagnóstico inicial.

La toma y procesamiento de muestra, amplificación y análisis de resultados fueron realizados en el Laboratorio de Virología y Microbiología Molecular del Departamento de Microbiología, Facultad de Medicina, Universidad de El Salvador.

\section{Secuenciación masiva}

De las muestras positivas se seleccionaron dos para ser secuenciadas por NGS en la plataforma MiniSeq Illumina con el kit de Paragon CleanPlex ${ }^{\oplus}$ SARS-CoV-2 by SOPHiA siguiendo el protocolo del fabricante. Para el alineamiento se utilizó la plataforma bioinformática SOPHiA-DDMV5.7.10.

\section{Consideraciones éticas}

Se cuenta con autorización del comité local de ética del Instituto Nacional de Salud. La base de datos codificada esta bajo el resguardo de la Sección de Virología del Departamento de Microbiología, Universidad de El Salvador. 


\section{Resultados}

Las muestras obtuvieron una concentración de RNA por fluorometría entre 50 a 90 ng/ $\mu \mathrm{l}$, fueron 8 muestras positivas para el gen E y $R d R p$ y 92 negativas para ambos genes. Tabla 1.

Tabla 1. Amplificación por RT-qPCR de muestras de saliva y secreción nasofaríngea

\begin{tabular}{|c|c|c|c|c|}
\hline \multirow[t]{2}{*}{$\begin{array}{l}\text { Volumen final con } 5 \mu \mathrm{l} \text { de } \\
\text { eluido }\end{array}$} & \multicolumn{2}{|c|}{ GEN E } & \multicolumn{2}{|c|}{$\begin{array}{l}\text { GEN } \\
\text { RdRp }\end{array}$} \\
\hline & 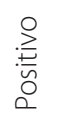 & $\begin{array}{l}\stackrel{0}{2} \\
\stackrel{D}{0} \\
\stackrel{D}{Z}\end{array}$ & 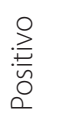 & $\begin{array}{l}\stackrel{0}{2} \\
\stackrel{1}{0} \\
\stackrel{D}{Z}\end{array}$ \\
\hline $\begin{array}{l}25 \mu \mathrm{l} \text {, Charité, Berlin/evag } \\
40 \text { ciclos }\end{array}$ & 8 & 92 & 8 & 92 \\
\hline $\begin{array}{l}20 \mu l \text {, Powerchek 2019- } \\
\text { ncov } 40 \text { ciclos }\end{array}$ & 8 & 92 & 8 & 92 \\
\hline
\end{tabular}

Las muestras positivas mostraron para el gen $E$ y $R d R p$ CT desde ciclo 18 hasta ciclo 35, el control positivo amplificó entre el ciclo 23 y 24 (Figura 1), todos los CT entre el gen E y RdRp fueron coincidentes, solo una de las muestras negativas mostró un CT no coincidente de 39 para el gen E en el protocolo PowerChek 2019-nCoV con CT no detectado para $R d R p$. Para esta misma muestra no se registró CT con protocolo Charité, Berlin / EVAg para ninguno de los genes, por lo quer se catalogó como negativa.

Las muestras positivas y sus alícuotas almacenadas $\mathrm{a}-80{ }^{\circ} \mathrm{C}$ se extrajeron y amplificaron manteniendo su concentración de ARN, todas se amplificaron en un CT similar al inicial en los dos protocolos con los genes E y $R d R p$ manteniendo su positividad al primero, tercero y quinto mes pos extracción inicial. De igual forma los eluídos positivos iniciales almacenadas $\mathrm{a}-80{ }^{\circ} \mathrm{C}$ se mantuvieron positivos al primero, tercero y quinto mes después del diagnóstico inicial.

Las dos secuencias obtenidas por NGS fueron positivas a SARS-CoV-2 en el análisis realizado en la plataforma SOPHiA-DDMV5.7.10. y fueron validadas y depositadas para su posterior análisis en la plataforma GISAID con registro: EPI_ISL_671974, EPI_ ISL_672573.

\section{Discusión}

El promedio de positivos por cada 100 pruebas en noviembre en El Salvador fue de 7,05 por cada 100 pruebas para COVID-19 realizadas con hisopado nasofaríngeo ${ }^{4}$, este dato es similar con el resultados de ocho pruebas positivas de 100 pruebas realizadas durante el mes de noviembre en el presente estudio, utilizando como muestra una mezcla de saliva y secreción faríngea espontanea o por carraspeo autotomada por el paciente utilizando tubos de polipropileno de $14 \mathrm{ml}$ vacíos y estériles (sin medio de transporte o SSN). Los protocolos de RT-qPCR utilizados (Charité, Berlin /EVAg y PowerChek 2019-nCoV) con los genes E y RdRp son los mismos utilizados en la mayoría de diagnósticos COVID-19 realizados por el Sistema Nacional de Salud, salvo por algunos equipos automatizados que utilizan gen $\mathrm{N}$. Adicionalmente se reconfirmó por NGS la positividad de las muestras. Estos resultados son coincidentes y respaldan los hallazgos obtenidos en otros estudios en los que se demuestra que la saliva y esputo nasofaríngeo, especialmente si es recolectada dentro de los 10 días posteriores al inicio de los síntomas, puede sustituir las muestras de frotis nasofaríngeos ${ }^{30-32}$ o que las muestras de saliva y las muestras de frotis nasofaríngeos tienen una sensibilidad similar en la detección del SARS-CoV-234.

El almacenamiento a largo plazo de las muestras de saliva y secreción nasofaríngea a $-80{ }^{\circ} \mathrm{C}$ no afectó los resultados de la prueba, incluso al $5^{\circ}$ mes posdiagnóstico. Esto sugiere que las muestras de saliva y secreción nasofaríngea recolectadas de los pacientes, pueden almacenarse a $-80{ }^{\circ} \mathrm{C}$ por meses sin perder sensibilidad lo que es coincidente con otros estudios ${ }^{30-32}$.

La autotoma de muestra de saliva tiene menor complejidad que la obtención del hisopado nasofaríngeo y esta técnica de autotoma de saliva requiere menor consumo de insumos y medidas de bioseguridad, solo se requiere abrir el tubo una sola ocasión para tomar los $200 \mu$ le muestra para el proceso de extracción ya que al no utilizar hisopo se elimina este paso de manipulación, este proceso ejecutado para diagnostico COVID-19 demuestra al igual que en otros estudios, que la saliva y secreción nasofaríngea autotomada por el paciente se convierte en una alternativa para el diagnóstico de la infección por SARS-CoV-2 $2^{31-33}$.

Si bien la concordancia de sensibilidad diagnostica de las pruebas es alta, el tipo de muestreo de la población en estudio no permite una comparación estadística, debido a que no se realizó hisopado nasofaríngeo simultáneo a las personas que participaron en el estudio por ser un estudio exploratorio. A pesar que el tiempo y recursos invertidos fueron registrados por los investigadores, estos serán sistematizados en estudios posteriores comparando los dos métodos (saliva-secreción nasofaríngea versus hisopado nasofaríngeo). Es necesario realizar estudios 


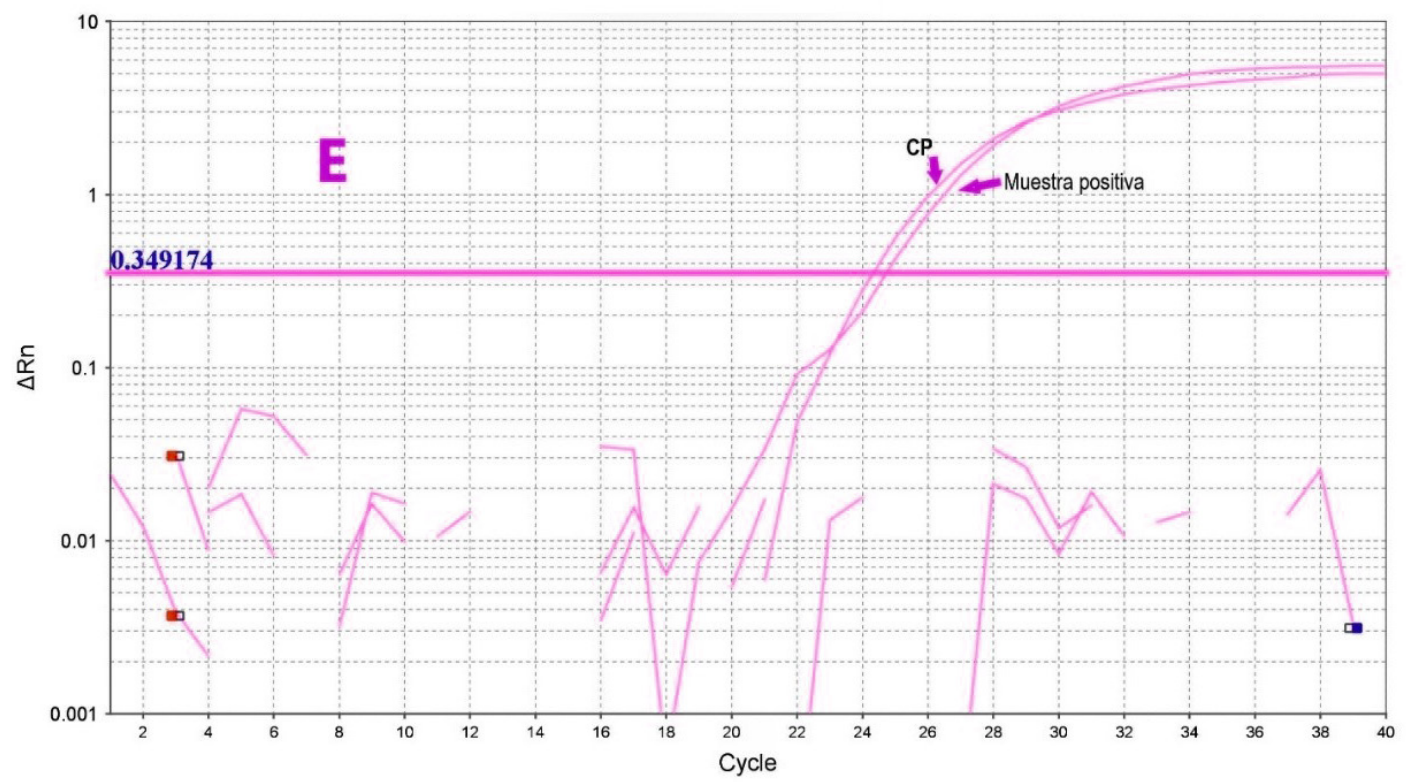

gen $\mathrm{E}$

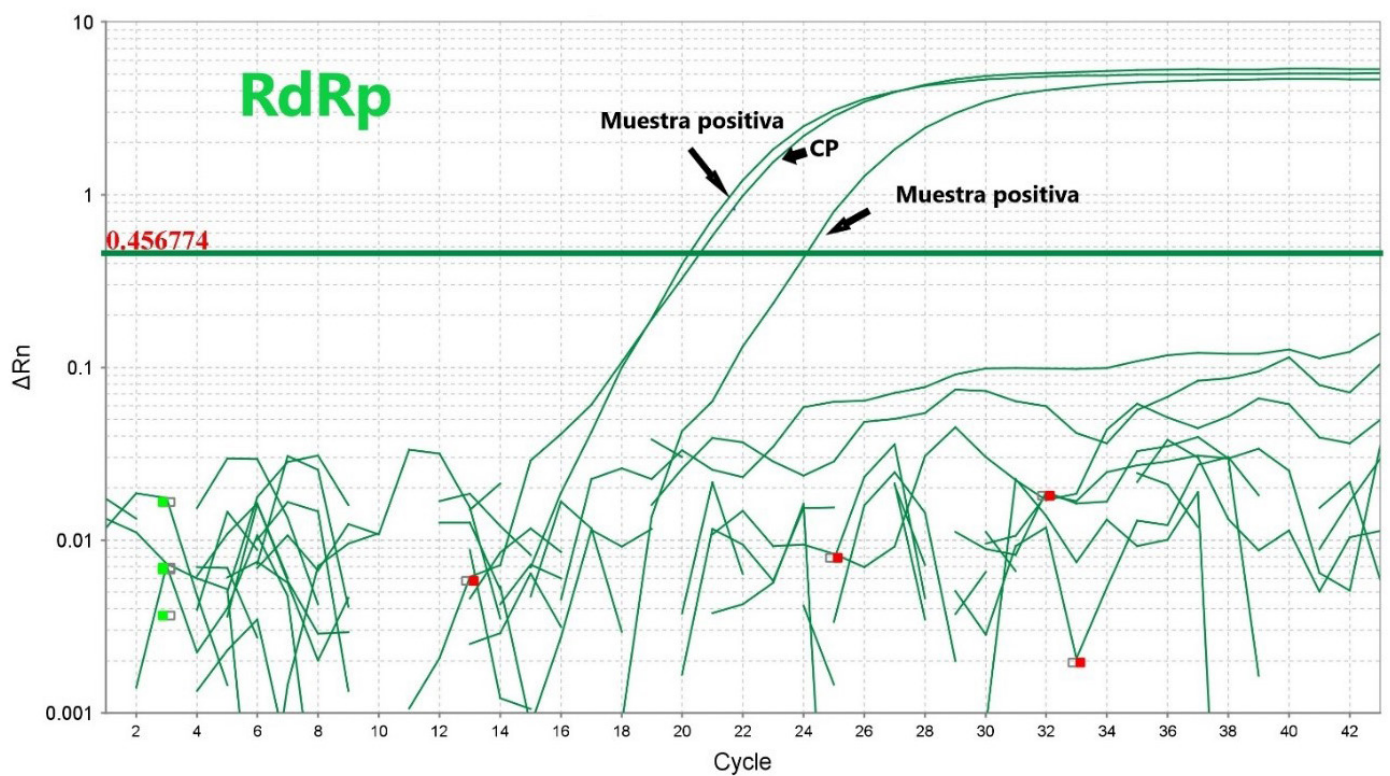

$\operatorname{RdRp}$

Figura 1. Curvas de amplificación de genes E y RdRp.

de costo-efectividad que permitan adoptar otras metodologías de detección de SARSCoV-2 y optimizar los recursos disponibles en nuestro país.

\section{Conclusiones}

La saliva y secreción nasofaríngea resulto efectiva para la detección de SARS-Cov-2 mediante la técnica de RT-qPCR a través de dos protocolos. Las medidas de bioseguridad para la toma de la muestra, como el distanciamiento y espacios ventilados son medidas de bioseguridad accesible para la mayoría de establecimientos de salud.

Las muestras positivas permanecieron viables para su diagnóstico hasta el quinto mes a $-80^{\circ} \mathrm{C}$ sin perder sensibilidad y especificidad. 
La utilidad de la muestra de saliva y secreción faríngea autotomada emitida de manera espontánea o carraspeo para el diagnóstico COVID-19 es una alternativa de menor complejidad al no utilizar hisopo, tubos de trasporte viral ni medios de conservación en el tubo. Es una medida no invasiva para el estudio de población sintomática ambulatoria o con exposición, útil para la detección de casos positivos a nivel comunitario, lugares de trabajo, escuelas y otros, reduciendo el riesgo del personal de salud que recolecta y procesa la muestra ya que no se expone a los aerosoles generados durante la toma de muestras con hisopo y se reduce la manipulación de la misma durante su análisis.

\section{Agradecimientos}

Se reconoce el apoyo de las autoridades de la Universidad de El Salvador, Instituto Nacional de Salud y Ministerio de Salud; agradecimientos especiales a la Sección de Virología del Departamento de Microbiología, Facultad de Medicina, Universidad de El Salvador y a la Sección de Virología del Laboratorio Nacional de Salud Pública de El Salvador.

\section{Referencias bibliográficas}

1. Su S, Wong G, Shi W, Liu J, Lai ACK, Zhou J, et al. Epidemiology, Genetic Recombination, and Pathogenesis of Coronaviruses. Trends Microbiol. 2016;24(6):490-502. DOI: 10.1016/j.tim.2016.03.003

2. Woo PCY, Lau SKP, Lam CSF, Lau CCY, Tsang AKL, Lau JHN, et al. Discovery of Seven Novel Mammalian and Avian Coronaviruses in the Genus Deltacoronavirus Supports Bat Coronaviruses as the Gene Source of Alphacoronavirus and Betacoronavirus and Avian Coronaviruses as the Gene Source of Gammacoronavirus and Deltacoronavi. J Virol. 2012;86(7):3995-4008. DOI: 10.1128/ JVI.06540-11

3. Wu F, Zhao S, Yu B, Chen YM, Wang W, Song $Z G$, et al. A new coronavirus associated with human respiratory disease in China. Nature. 2020;579(7798):265-9. DOI: 10.1038/s41586020-2008-3

4. Gobierno de El Salvador. Situación Nacional COVID-19. Gobierno de El Salvador. 2021. Fecha de consulta: marzo de 2021. Disponible en: https://covid19.gob.sv/

5. Organización Mundial de la Salud. Pruebas diagnósticas para el SARSCoV-2: orientaciones provisionales. Ginebra. Organización Mundial de la Salud. 11 de septiembre de 2020. 26 p.
Disponible en: https://apps.who.int/iris/ handle/10665/335830.

6. John Hopkins University Coronavirus Resource Center. COVID-19 dashboard by the Center for Systems Science and Engineering (CSSE) at Johns Hopkins University. John Hopkins University. 2021. Fecha de consulta: marzo 2021. disponible en: $\underline{\text { https:// }}$ www.arcgis.com/apps/dashboards/ bda7594740fd40299423467b48e9ecf6

7. World Health Organization. WHO Coronavirus (COVID-19) Dashboard. World Health Organization. 2021. Switzerland. Fecha de consulta: marzo 2021. Disponible en: https://covid19.who.int/

8. World Health Organization. Draft landscape and tracker of COVID-19 candidate vaccines. WHO. 2021. Fecha de consulta: marzo 2021 Disponible en: https://www.who.int/ publications/m/item/draft-landscape-ofcovid-19-candidate-vaccines

9. Randolph HE, Barreiro LB. Herd Immunity: Understanding COVID-19. Immunity. 2020;52(5):737-41. DOl: https://doi. org/10.1016/j.immuni.2020.04.012

10. ElBagoury M, Tolba MM, Nasser HA, Jabbar A Hutchinson A. The find of COVID-19 vaccine: Challenges and opportunities. J Infect Public Health. 2020;14(3):389-416. DOl: https://doi. org/10.1016/j.jiph.2020.12.025

11. World Health Organization. WHO concept for fair access and equitable allocation of COVID-19 health products. Geneva. WHO. 9 September 2020. 34 p. Disponible en: https://www.who.int/publications/m/item/ fair-allocation-mechanism-for-covid-19vaccines-through-the-covax-facility

12. Herzog LM, Norheim OF, Emanuel EJ, McCoy MS. Covax must go beyond proportional allocation of covid vaccines to ensure fair and equitable access. BMJ. 2021;5;372:m4853. DOI: 10.1136/bmj.m4853.

13. Su S, Wong G, Shi W, Liu J, Lai ACK, Zhou J, et al. Epidemiology, Genetic Recombination, and Pathogenesis of Coronaviruses. Trends Microbiol . 2016;24(6):490-502. DOI:10.1016/j.tim.2016.03.003

14. Chen Y, Liu Q, Guo D. Emerging coronaviruses: Genome structure, replication, and pathogenesis. J Med Virol. 2020;92(4):418-23. Disponible en: https:// pubmed.ncbi.nlm.nih.gov/31967327/

15. Gordon DE, Jang GM, Bouhaddou $M, X u$ J, Obernier K, White KM, et al. A SARS$\mathrm{CoV}-2$ protein interaction map reveals targets for drug repurposing. Nature. 2020;583(7816):459-68. DOI: 10.1002/ jmv. 25681

16. Hernandez Avila CE, Ortega 'Perez CA, Rivera NR, López XS. Análisis de la mutación D614G encontrada en secuencias del genoma completo de SARS-CoV-2 en El Salvador. 
Alerta. 2021;4(1):72-7. DOl: 10.5377/alerta. v4i1.10683

17. Kim D, Lee JY, Yang JS, Kim JW, Kim VN, Chang $\mathrm{H}$. The Architecture of SARS-CoV-2 Transcriptome. Cell. 2020;181(4):914-921. e10. DOI: 10.1016/j.cell.2020.04.011

18. Stegeman I, Ochodo EA, Guleid F, Holtman GA, Yang B, Davenport C, et al. Routine laboratory testing to determine if a patient has COVID-19 Cochrane Infectious Diseases Group, editor. Cochrane Database Syst. Rev. 2020;11:1465-1858. DOI:10.1002/14651858. CD013787

19. Sethuraman N, Jeremiah SS, Ryo A. Interpreting Diagnostic Tests for SARSCoV-2. JAMA. 2020;323(22):2249. DOI:10.1001/jama.2020.8259

20. Reina J, Suarez L. Evaluation of different genes in the RT-PCR detection of SARS$\mathrm{CoV}-2$ in respiratory samples and its evolution in infection. Rev. Esp. Quimioter. 2020;33(4):292-293. DOI: 10.37201/ req/045.2020

21. Pinilla G, Cruz B CA, Navarrete O J. Diagnóstico molecular de SARSCoV-2. Nova. 2020;18(35):35-41. DOI: 10.22490/24629448.4184

22. Wölfel R, Corman VM, Guggemos W, Seilmaier M, Zange S, Müller MA, et al. Virological assessment of hospitalized patients with COVID-2019. Nature. 2020;581(7809):465-469. DOI: 10.1038/ s41586-020-2196-x

23. Leung EC, Chow VC, Lee MK, Lai RW. Deep throat saliva as an alternative diagnostic specimen type for the detection of SARSCoV-2. J. Med. Virol. 2021;93(1):533-536. DOI: $10.1002 / j m v .26258$

24. Takeuchi Y, Furuchi M, Kamimoto A, Honda K, Matsumura H, Kobayashi R. Saliva-based PCR tests for SARS-CoV-2 detection. J. Oral Sci. 2020;62(3):350-351. DOI: 10.2334/ josnusd.20-0267

25. Fakheran O, Dehghannejad M, Khademi A. Saliva as a diagnostic specimen for detection of SARS-CoV-2 in suspected patients: a scoping review. Infect. Dis. Poverty. 2020;9(1):100. DOI: 10.1186/s40249020-00728-w

26. Williams E, Isles N, Chong B, Bond K, Yoga Y, Druce J, et al. Detection of SARS-CoV-2 in saliva: implications for specimen transport and storage. J. Med. Microbiol. 2021;70(2). DOI:10.1099/jmm.0.001285

27. Ben-Assa N, Naddaf R, Gefen T, Capucha T, Hajjo H, Mandelbaum N, et al. Direct on-thespot detection of SARS-CoV-2 in patients.
Exp. Biol. Med. 2020;245(14):1187-1193.

DOI: 10.1177/1535370220941819

28. Butler-Laporte G, Lawandi A, Schiller I, Yao M, Dendukuri N, McDonald EG, et al. Comparison of Saliva and Nasopharyngeal Swab Nucleic Acid Amplification Testing for Detection of SARS-CoV-2: A Systematic Review and Meta-analysis. JAMA Intern. Med. 2021;181(3):353. DOI: 10.1001/ jamainternmed.2020.8876

29. Pasomsub E, Watcharananan SP, Boonyawat K, Janchompoo P, Wongtabtim G, Suksuwan W, et al. Saliva sample as a non-invasive specimen for the diagnosis of coronavirus disease 2019: a cross-sectional study. Clin. Microbiol. Infect. 2021;27(2):285.e1-285.e4. DOI: 10.1016/j.cmi.2020.05.001

30. Uwamino Y, Nagata M, Aoki W, Fujimori Y, Nakagawa T, Yokota H, S, et al. Accuracy and stability of saliva as a sample for reverse transcription PCR detection of SARS-CoV-2. J. Clin. Pathol. 2021;74(1):67-68. DOI: 10.1136/jclinpath-2020-206972

31. Pasomsub E, Watcharananan SP, Watthanachockchai T, Rakmanee K, Tassaneetrithep B, Kiertiburanakul S, et al. Saliva sample pooling for the detection of SARS-CoV-2. J. Med. Virol. 2021;93(3):15061511. DOI: 10.1002/jmv.26460

32. Ceron J, Lamy E, Martinez-Subiela S, LopezJornet P, Capela-Silva F, Eckersall P, et al. Use of Saliva for Diagnosis and Monitoring the SARS-CoV-2: A General Perspective. J. Clin. Med. 2020;9(5):1491. DOI: 10.3390/ jcm9051491

33. Azzi L, Maurino V, Baj A, Dani M, d'Aiuto A, Fasano M, et al. Diagnostic Salivary Tests for SARS-CoV-2. J. Dent. Res. 2021;100(2):115123. DOI: $10.1177 / 0022034520969670$

34. Wyllie AL, Fournier J, Casanovas-Massana A, Campbell M, Tokuyama M, Vijayakumar P, et al. Saliva or Nasopharyngeal Swab Specimens for Detection of SARS-CoV-2. N. Engl. J. Med. 2020;383(13):1283-1286. DOI: 10.1056/NEJMc2016359

35. Kampf G, Voss A, Scheithauer S. Inactivation of coronaviruses by heat. J Hosp Infect. 2020;105(2):348-9. Available from: https:// doi.org/10.1016/j.jhin.2020.03.025

36. Rabenau HF, Cinatl J, Morgenstern B, Bauer G, Preiser W, Doerr HW. Stability and inactivation of SARS coronavirus. Med. Microbiol. Immunol. (Berl.). 2005;194(1-2):16. DOI: 10.1007/s00430-004-0219-0 